year period. While accounting for surgical BMI and age, quantile regression was used to create expected WL curves (10th, 25th, 50th, 75th, and 90th \%tile) for the 36 month post-operative period. Results: A mobile application (Get-2-Goal) was designed to provide a simple, personalized interface that allows patients to track their WL and compare their WL results to their expected WL curves. Get-2-Goal was made publicly available at no cost on a popular Apps store and is compatible with current smartphone and tablet technology ( $>1000$ downloads to date). Get-2-Goal allows patients to input their personal profile (e.g. age, BMI), review their expected WL, and track their WL post-operatively. Patients have the option of saving a graphic containing their personalized WL curves and e-mailing a tabular form of their WL results to family, friends, and/or care providers. Conclusions: Get-2Goal is a simple tool that may be used by Bariatric Surgery Programs to facilitate electronic patient education. This tool may assist patients in deciding to proceed to WL surgery, and will facilitate early identification of patients who are struggling with WL or weight maintenance. Future modifications of this methodology can be used to personalize other comorbidity outcomes such as diabetes resolution.

Keywords: Patient-centered; Bariatric surgery

$$
\text { doi:10.3121/cmr.2014.1250.ps1-48 }
$$

\section{Embedded Research}

A3-1:

The Just Do It Playbook for Implementation Science

Adam Sharp ${ }^{1}$; Huong Nguyen'; Erin Hahn ${ }^{1}$; Tania Tang${ }^{1}$; Brian Mittman ${ }^{1}$; Michael Kanter ${ }^{1}$; Steve Jacobsen ${ }^{1}$; Michael Gould ${ }^{1}$

\section{${ }^{1}$ Kaiser Permanente Southern California}

Background/Aims: There are over 60 published implementation science frameworks, and despite the advantages of each, none were designed to help clinicians and administrators to standardize and prioritize initiatives, implement programs efficiently, and evaluate with sufficient rigor. Methods: In order to address these limitations, the Care Improvement Research Team (CIRT) at Kaiser Permanente Southern California blended implementation and behavior change frameworks into a structured process to help clinicians, operations leaders and researchers to standardize their approach and accomplish collaborative goals. To develop the "Just Do It Playbook," we performed a pragmatic literature review, compared and synthesized published frameworks, and iteratively refined a set of steps for identifying, diagnosing and correcting gaps in care. Results: The Just Do It Playbook can be simplified into four distinct steps: 1) describe the topic that needs improvement, 2) identify what or who needs to change, 3) develop an implementation strategy to facilitate change, and 4) evaluate the effectiveness of the strategy. The first step helps to prioritize the topic of interest and identifies whether there is a gap between current and best practices. Second, describing what or who needs to improve will enable identification of barriers or facilitators to change. The third step identifies strategies to promote best practices by using established behavior change theories as well as clearly defined and measurable outcomes. The final step evaluates the outcomes, costs and sustainability of the strategy, as well as the need to discontinue efforts, modify and re-evaluate, or disseminate to other settings. Conclusions: In a learning health care system, there are many potential targets for improvement, and many strategies for implementation and evaluation. One way to simplify the complexities that inherently exist in health system improvement is to adopt a standardized approach. As clinicians, administrators and researchers forge meaningful collaborations, a standard process can improve the understanding of competing priorities, define roles and responsibilities, and integrate established scientific frameworks in a way that is efficient and sustainable. The Just Do It Playbook will need to be tested and adapted to individual projects, but will provide a platform to build embedded research into a functional operational standard.

Keywords: Research-operations partnership; Implementation science doi:10.3121/cmr.2014.1250.a3-1
A3-2:

Mindful Inquiry: From an Observer to a Gentle Change Agent in the Prevailing Culture of Do and Tell

Ming Tai-Seale ${ }^{1}$

${ }^{1}$ Palo Alto Medical Foundation Research Institute

Background/Aims: In a culture of do and tell, researchers can use massive amount of EHR data, find statistically significant results, and publish them, often without the need to interact with the people who generated those data through their daily work or their use of health care services. Such observational inquiry can be informative one hand, as identifying areas for improvement requires the identification of gaps in quality of care or other shortcomings in the system. Mindful inquiry, i.e., asking questions in the moment, the here-and-now, on the other hand, has the potential to become a change agent that can facilitate the creation of new knowledge and the dissemination and implementation of best practices. Methods: Appreciative inquiry. Results: Task oriented culture of research values doing more than relating, telling more than asking, thereby leaving room for improvement in our capacity and desire to form and keep relationships with our research subjects or the organization where we do research. The practice of fly-bynight researcher who swoops down, collects data, publishes papers that expose dark sides of the subjects' lived world has caused permanent harm to communities. Embedded researchers need to be mindful about the questions they raise, the methods they employ, and how they disseminate the results. Conclusions: Embedded research is a series of complex, interdependent tasks which requires building positive, trusting relationships with research team and delivery system partners to facilitate good communication. We must be better at asking and do less at telling. Transform our mindset from the researcher knows a lot to the practitioners know a lot. We must be open to adaptive learning, be curious and courageous enough to ask questions to which we do not already know the answer. Good communication is key to building a trusting relationship which requires mindful inquiry, done with humility to build positive relationships with the delivery organization and guided by the wish to be of service to the greater good.

Keywords: Embedded research; Change agent

doi:10.3121/cmr.2014.1250.a3-2

\section{A3-4:}

Implementing a Pragmatic Clinical Trial: Lessons Learned about Embedded Research

Lindsay Kindler'; Carmit McMullen ${ }^{1}$; Ashli Owen-Smith²; Stacey Honda ${ }^{3}$; Alison Firemark ${ }^{1}$; Lynn DeBar ${ }^{1}$

${ }^{1}$ Kaiser Permanente Northwest; ${ }^{2}$ Kaiser Permanente Southeast; ${ }^{3}$ Kaiser Permanente Hawaii

Background/Aims: Pragmatic trials are fostering a paradigm shift to increasingly embed research into healthcare settings. Pragmatic trials test interventions in real world settings representative of those targeted for dissemination to promote robust, sustainable changes within the health care system. Kaiser Permanente's Center for Health Research, is conducting a pragmatic trial to test the effectiveness of teaching pain self-management skills using interdisciplinary teams within primary care in three Kaiser regions. Implementation requires a pragmatic approach to create a working relationship between health system leaders and the research team. We present lessons learned during our preparatory year in which we partnered with health systems to build an embedded research infrastructure for the study. Methods: In accordance with a pragmatic approach, we used the Rapid Assessment Process (RAP) whereby the fieldwork team gathers and analyzes information quickly in the form of journal entries and detailed meeting minutes following stakeholder encounters and regular team meetings to document the emerging collective understanding of stakeholders' expressed concerns and informational needs. This approach allows for iterative feedback from operational stakeholders and accommodates quick trial modifications. Results: Using RAP to systematically identify lessons learned, several guiding principles surfaced for successfully embedding research into a healthcare system. The study foundation must come from a need identified by the health system, not the research team. Study outcomes must have obvious utility to operational leaders and practicing providers. 
Health plan administrators and clinicians have pre-existing ideas of research that necessitate ongoing education and dialogue to address concerns. Using a framework of change native to the health system promotes navigation of potentially disruptive change. Given rapid changes within health systems, the research team must be aware of competing contextual factors. Finally, a true health system/research partnership when staffing the intervention facilitates sustainability of intervention related services. Conclusions: A pragmatic trial approach, although better suited for embedded research in the healthcare system, presents challenges not typically encountered in standard explanatory/ efficacy trials. Lessons learned by this team that accommodate both healthcare operations and research can promote embedded research in other health settings.

Keywords: Pragmatic clinical trial; Chronic pain

doi:10.3121/cmr.2014.1250.a3-4

\section{A3-5: \\ Research-Operations Partnerships to Improve the Quality and Affordability of Care}

Michael Gould ${ }^{1}$; Huong Nguyen ${ }^{1}$; Adam Sharp ${ }^{1}$; Erin Hahn'; Tania Tang ${ }^{1}$; Brian Mittman ${ }^{1}$; Steven Jacobsen ${ }^{1}$; Michael Kanter ${ }^{1}$

\section{${ }^{1}$ Kaiser Permanente Southern California}

Background/Aims: A learning health care organization requires visionary leadership to achieve the triple aim of better health, better care, and improved affordability. Effective research-operations partnerships (ROPs) can facilitate improvement efforts. Although ROPs are not novel, the process of establishing sustainable collaborations and overcoming challenges to achieve shared objectives is not well described. Methods: In 2012, the executive leadership of Kaiser Permanente Southern California (KPSC) made a multi-million dollar investment by creating the Care Improvement Research Team (CIRT) within the Department of Research and Evaluation. Members of this team developed and refined ideas about ROPs by reviewing published literature, conducting semi-structured interviews with key operational leaders, consulting with experts, and sponsoring an all-day retreat on this theme. Results: Key elements of the initiative include: (1) recruitment of health care researchers with complementary clinical and methodological experience, supported by an expert consultant in implementation science; (2) selection of research questions that are clearly aligned with organizational priorities; (3) development of strong, sustainable relationships with key stakeholders across multiple levels of the organization, through outreach and embedding of researchers in operational work groups; (4) dedication of internal funds to cover effort of research support staff; (5) creation of a strategic plan to define roles, responsibilities, and goals that allow for measurement of the program's success. Key challenges to overcome include: (1) limited availability of external funds to support research that addresses operational imperatives; (2) mismatched timelines and incentives; (3) legal constraints that hamper data sharing between research and operations; and (4) balancing research rigor with operational relevance. Conclusions: Prioritizing competing initiatives, identifying opportunities for early success, and aligning operational priorities with research opportunities will require continued effort. Given the austere external funding environment, novel ways to support delivery system science are needed. The creation of the CIRT at KPSC represents a strategic investment from visionary leaders who embody the credo to "be the best at getting better" and who recognize that the development of strong and sustainable ROPs will be invaluable if we are to achieve the triple aim.

Keywords: Partnerships; Care improvement

doi:10.3121/cmr.2014.1250.a3-5

PS1-20:

Understanding Patient Barriers and Preferences to Completing Advance Directives (AD) in the Primary Care Setting

J.B. Jones ${ }^{1}$; Janet Tomcavage ${ }^{1}$; Dorothy Fisher ${ }^{2}$; Ryan Van Loan ${ }^{1}$; Virginia Lerch $^{1}$; Thomas Graf ${ }^{1}$

\section{Geisinger Health System; ${ }^{2} \mathrm{New}$ West Health Services}

Background/Aims: Advance Care Planning (ACP) is a complex process that allows individuals to contemplate and document end of life decisions using tools such as an Advance Directive (AD). The proportion of patients who have an $\mathrm{AD}$ on file remains low both nationally and at Geisinger. To date, little research has focused on healthy populations' attitudes towards ACP and AD completion. We describe the design and implementation of a web-based application to collect patients' preferences for and barriers to $\mathrm{AD}$ completion in a large, non-diseased primary care population. Methods: We developed a simple web application and questionnaire (denoted "eACP") designed to educate patients about completing an AD. The eACP application was automatically presented on a touchscreen computer to all patients aged 50-64 who were seen in one of 5 Geisinger Clinic locations for a routine appointment. The questionnaire introduced ACP as a part of good healthcare and asked patients if they were interested in learning more. Patients who chose not to learn more indicated why they declined. Patients who elected to learn more selected topics of $\mathrm{AD}$ completion for which they would like more information and indicated how they wished to review the information. Results: A total of 2169 patients completed the questionnaire using the eACP application in 5 practice sites between $07 / 31 / 13$ and 10/30/13. Nearly $40 \%$ $(852 / 2169)$ of patients were interested in learning more while $49.8 \%$ (1080/2169) were not. The primary reasons for declining to learn more included lack of time, a preference for leaving the choice to others, or prior $\mathrm{AD}$ completion. Among the patients who elected to learn more, the most common topics of interest were related to the process of completing an $\mathrm{AD}$ (e.g., what goes into an $\mathrm{AD}$ and how/when to complete it). Patients had a strong preference for printed materials $(70 \%)$ versus using a website $(30 \%)$ or talking to a healthcare professional $(<10 \%)$. Conclusions: Our findings suggest that patients desire more education on ADs but prefer to receive it in a paper format versus online or via a discussion with their provider. Strategies for increasing AD completion in practice should account for these patient preferences.

Keywords: Patient-reported data; Advance care planning

doi:10.3121/cmr.2014.1250.ps1-20

PS1-21:

Development and Dissemination of Quality Review Tools for Data Management and Analysis

Sophia Newcomer ${ }^{1}$; Liza Reifler ${ }^{1}$; Jennifer Ellis ${ }^{1}$

${ }^{1}$ Kaiser Permanente Colorado

Background/Aims: Within embedded HMO research groups, analytic teams are heavily involved with many key stages of research studies. Individual analytic team members use a variety of processes for ensuring work quality. Identification and standardization of analytic quality review best practices may lead to greater efficiencies and fewer errors. The objective of this quality improvement project was to develop and implement tools to enhance the quality and consistency of analytic work within a HMO-based research group. Methods: We used a multi-modal approach to develop and assess analytic quality review tools. We first conducted four in-depth interviews with principal investigators to collect experiences and suggestions for improving analytic processes. Using an online survey, we collected best practices from analytic team members. We also interviewed analytic leads with $6 \mathrm{HMO}$ and academic partner research groups on their best practices. We integrated all information and developed analytic quality review best practice documentation and tools. We tested and revised the tools, estimated time requirements, and provided basic training to our analytic team. Nine months later, we conducted an anonymous online survey to gauge uptake of these tools and to collect initial feedback from early adopters. Results: Four analytic quality review tools were developed to help formalize best practices for cohort-building/data pulls, data preparation (code review), analytic dataset preparation and documentation, and manuscript methods and results review. In initial assessments, implementing the code review was the most resource-intensive, taking upwards of 14 hours. Nine months after introducing these tools, the most commonly reported challenges to using the tools included lack of time/funding, using other processes to ensure work quality, and lack of knowledge on when or how to use the tools. Early adopters gave qualitative feedback that the tools helped structure analytic processes and encouraged documentation of analytic decisions. Conclusions: Identification and standardization of best practices may have the potential to improve analytic work processes for HMO research groups. Future efforts should focus on quality review tool revisions, policies for tool use, data collection 